\title{
The impact of steroids on hearing after bacterial meningitis
}

Lauren Sorce RN, MSN, CCRN, CPNP-AC/PC, FCCM, Pediatric Nurse Practitioner, Pediatric Critical Care, Children's Memorial Hospital, Chicago, IL USA

Key Words: hearing loss * meningitis paediatrics steroids *

E-mail: Isorce@childrensmemorial.org

\section{ESPAÑOL}

Impacto de los esteroides en la audición después de la meningitis bacteriana

\section{Palabras clave}

Esteroides, meningitis, pediatría, pérdida de audición

Resumen

La meningitis puede ser una enfermedad agobiante en pediatría.

El tratamiento de la meningitis depende de la etiología, el cuidado de sostén es crucial para limitar los efectos devastadores de la enfermedad.

Una medida terapéutica ha sido el uso de esteroides para disminuir el riesgo de desarrollar la pérdida de audición neurosensorial.

\section{SUMMARY}

- Meningitis can be a crippling disease in pediatrics.

- Treatment of meningitis is dependent on the aetiology and supportive care is crucial to limit the devastating effects of this disease.

- One therapeutic approach has been the use of steroids to decrease the risk of development of sensorineural hearing loss.

\section{INTRODUCTION}

Across the world, meningitis remains a debilitating disease of children. Depending on the geographical region, epidemiology varies. However, bacterial meningitis continues as a devastating disease. This article will review the use of steroids as an adjunctive therapy to reduce the risk of hearing loss associated with bacterial meningitis.

\section{THE DISEASE}

Bacterial meningitis is an inflammation of the covering of the brain and spinal cord. It affects the pia, arachnoid and subarachoid space. The involved pathogen enters the brain through the blood brain barrier. A variety of chemical cascades are initiated, depending on the pathogen, which the result in inflammation.
Signs and symptoms of the disease vary somewhat based on the age of the child. Infants may have fever or hypothermia, irritability, bulging fontanel, poor feeding, lethargy, respiratory distress, vomiting, or seizures. Children may present with fever, irritability, confusion, seizures, stiff neck, photophobia, nausea/vomiting, and altered mental status.

On physical exam, one may identify Brudzinski's sign (when the neck is passively flexed, the hips flex), Kernig's sign (hip flexion and extension of the knee results in pain in the posterior trunk and legs), signs of increased intracranial pressure and/or focal neurological signs. Not all children will present with signs of meningeal irritation (Kernig's and Brudzinski's signs), so one should not rule out meningitis if these signs are not present. If Cushing's triad is present (hypertension, bradycardia and apnea), the child has advanced increased intracranial pressure and emergent measures should be taken to reduce it.

Meningitis is diagnosed using clinical and laboratory findings. Most patients undergo a lumbar puncture to obtain cerebrospinal fluid for cell count, gram stain and culture of the pathogen. Once the diagnosis is made, directed antibiotic therapy for the bacterial pathogen ensues. Additionally, therapies to reduce intracranial pressure should be used for evidence of intracranial hypertension.

\section{Hearing Loss}

Hearing loss secondary to meningitis was described in the 1920's by Shambaugh and colleagues (Shambaugh, Hagens \& Watkins, 1928). In a study of children attending schools for the hearing impaired, they identified meningitis as the link to acquired deafness in $17.5 \%$ of the children. They stated that the meningitis may be associated with an extension of the infection leading to otitis media which results in damage to the vestibular and acoustic mechanisms. However, if the infecting agent penetrated the labyrinth, the vestibular mechanism may or may not be damaged (Shambaugh, 1928). Decades later, it is known that hearing impairment is secondary to the infectious and inflammatory process associated with bacterial meningitis. In fact, the incidence of hearing loss associated with bacterial meningitis is around $9 \%$, depending on the study reviewed. Also, bacterial meninigitis is cited as being the largest cause of sensorineural hearing loss (SNHL) in children (Nadol, 1978).

Depending on the infectious pathogen, the pathophysiology of sensorineural hearing SNHL varies. Du, Wu and Li (2006) present a succinct look at the mechanisms of SNHL secondary to bacterial meningitis. They review the following processes: suppurative labyrinthitis and ossification; cytokine proliferation leading to alteration in the blood-brain barrier; and the role of reactive oxygen and nitrogen species. They also conclude that most of the time, the hearing loss is due to injury in the cochlea (Du, Wu \& Li, 2006). 
Determining the risk for development of $\mathrm{SNHL}$ is important when faced with a child with meningitis. de Jonge and colleagues (2010) studied risk factors associated with negative outcomes after bacterial meningitis. In the systematic review, meninigitis caused by streptococcus pneumoniae pathogen combined with a low glucose in the cerebrospinal fluid (CSF) were risk factors associated with the development of SNHL (de Jonge et al., 2010). While other studies have cited risks of time to provision of care, CSF glucose and streptococcus pneumoniae held up in this systematic review.

\section{STEROIDS}

Given that the pathophysiology of SNHL involves activation of the inflammatory cascade, it makes sense that use of a powerful anti-inflammatory agent (steroids) would be effective against the incidence of this meningitis-related outcome. Numerous studies have evaluated the usefulness of steroids in the treatment of bacterial meningitis, however, only a few will be discussed here

One of the first studies was done by deLemos and Haggerty (1969). They enrolled children greater than 1 month of age diagnosed with bacterial meningitis. Each patient had a lumbar puncture and gram stain done and was subsequently started on antibiotics. Each patient was then randomly assigned to receive placebo or methylprednisolone every 6 hours for 3 days. Upon evaluation, there was no significant improvement in mortality, morbidity or neurological outcomes between children who received steroids and those who did not. However, the resolution of fever was faster in the steroid group versus the placebo group (52.6hours compared to 70 hours). This study did not specifically evaluate the incidence of SNHL.

In 1988, Lebel and colleagues studied antibiotic therapies delivered with 4 days of dexamethasone or placebo. In this study of 200 subjects, the subjects who received antibiotics in addition to dexamethasone were less likely to have acquired moderate or severe hearing loss (Lebel et al., 1988). In 1993, Schaad and colleagues evaluated the use of dexamethasone given 10 minutes prior to antibiotics in 115 children in Switzerland (Schaad et al., 1993). Five percent of the children treated with dexamethasone in this study had hearing deficits compared to $16 \%$ of those who did not receive dexamethasone. While this was not statistically significant, there was a trend towards significance.

More than 15 years later, Peltola and colleagues (2009) completed a randomized, double blind study of 383 children diagnosed with bacterial meninigitis. Children were given ceftriaxone and assigned to 1 of 4 treatment groups: dexamethasone and placebo; dexamethasone and glycerol; glycerol and placebo; placebo and placebo. Upon completion of the study, there was no difference in hearing loss with any combination of dexamethasone, glycerol and/ or placebo at hearing loss levels of 40,60 and 80 decibels. While this study does not support the prior discussed outcomes, it is nonetheless helpful when trying to determine whether or not steroids should be administered to children who have bacterial meninigitis.

As a result of conflicting study results administering dexamethasone to children who have bacterial meningitis, Mclntyre, Mclntyre, Gilmour and Wang (2005) performed a population based study on the impact of steroid on meningitis. They discovered that in addition to early recognition of pneumococcal meningitis, adjunctive therapy with dexamethasone was a statistically significant predictor of improved neurological outcomes which included hearing loss.

In 2010, van de Beek and colleagues performed a meta-analysis of individual patients to determine which patient groups responded positively to the administration of dexamethasone in bacterial meninigitis. In an extensive meta-analysis including 2029 subjects from Europe, Malawi, Vietnam and South America, there was no difference in hearing outcomes for those patients treated with dexamethasone compared to those who did not receive it. It was only on post hoc analysis, when combined with older published trials, that the improvement in hearing outcomes were statistically significant when dexamethasone was administered (van de Beek et al., 2010).

Because the impact of this therapy has significant potential to help or not help children, there has been a Cochrane review of the administration of steroids to children with bacterial meningitis (Brouwer et al., 2010). The review supports the use of corticosteroids to reduce hearing loss and overall neurological sequelae. However, there was no impact on overall mortality. Additionally, it is recommended that this medication be used in high income countries but the data does not support the use of steroids in low income countries. Perhaps this is related to poor access to health care services in a timely manner to impact the overall therapy for bacterial meninigitis.

In summary, bacterial meningitis remains a devastating illness for children. Any attempt to modulate hearing outcomes is potentially helpful, including the use of steroids. While the data remains somewhat controversial on whether or not it decreases the incidence of SNHL, the administration of dexamethasone did not have negative outcomes associated with it. Therefore, as we know it is not going to harm the child, perhaps it is worth it to administer it in the event it will impact a change.

\section{REFERENCES}

Brouwer MC, McIntyre P, de Gans J, Prasad K, van de Beek D (2010). Corticosteroids for acute bacterial meninigitis. The Cochrane Database of Systematic Reviews, 9. DOI: 10.1002/14651858.CD004405.pub3.

DeLemos RA, Haggerty RJ (1969). Coprticosteroids as an adjunct to treatment in bacterial meningitis a controlled clinical trial. Pediatrics 44(1), 30-34.

De Jonge RCJ, van Furth AM, Wassenaar M, Gemke RJBJ, Terwee CB (2010). Predicting sequelae and death after bacterial meningitis in childhood: a systematic review of prognostic studies. BMC Infectious Diseases 10, 232.

Du Y, Wu X, Li L (2006) Mechanisms of bacterial meningitis-related deafness. Drug Discovery Today 3(1), 115-118.

Lebel MH, Freij BJ, Syrongiannopoulos GA, Chrane DF, Hoyt MJ, Stewart SM, Kennard BD, Olsen KD, McCracken GH Jr (1988). Dexamethasone therapy for bacterial meningitis. Results of two double-blind, placebo-controlled trials. NEJM 319, 964-971.

McIntyre PB, Mclntyre CR, Gilmour R, Wang H (2005). A population based study of the impact of corticosteroid therapy and delayed diagnosis on the outcome of childhood pneumococcal meningitis. Archives of Diseases of Childhood 90, 391-396.

Nadol JB, Jr (1978). Hearing loss as a sequela of meningitis. Laryngoscope 88(5), 739-55.

Schaumbaugh GE, Hagens EW, Watkins, RW (1928). Physical causes of deafness, II. Statistical studies of children in the public schools for the deaf. Archives of Otolaryngology 7(5), 424-513.

Van de Beek D Farrar J, de Gans J, Hoang NT, Molyneux EM, Peltola $\mathrm{H}$ et al (2010). Adjuntive dexamethasone in bacterial meningitis: a meta-analysis of individual patient data. Lancet Neurology 9, 254-263. 